\title{
Design considerations for early-phase clinical trials of immune-oncology agents
}

\section{Background}

The advent of the breakthrough therapy designation for experimental drugs by the Food and Drug Administration (FDA), initiated as part of the FDA Safety and Innovation Act (FDASIA) in July 2012, has taken oncology drug development in a new direction. A therapy receives this FDA designation if it is "one which is intended alone or in combination to treat a serious or life-threatening disease or condition, and for which preliminary clinical evidence indicates the drug may demonstrate substantial improvement over existing therapies on one or more clinically significant endpoints" [1, 2]. Such a designation from the FDA accelerates the review and development process of the new therapy, prompting regular communication

\footnotetext{
* Correspondence: nwages@virginia.edu

1Division of Translational Research \& Applied Statistics, Department of Public Health Sciences, University of Virginia, P.O. Box 800717, Charlottesville, VA, USA

Full list of author information is available at the end of the article
}

between the FDA and the sponsor to guide the development of promising agents. Since the FDASIA was signed into law, there have been several accelerated approvals of cancer drugs, particularly immune-oncology agents. For example, the immune checkpoint inhibitor pembrolizumab was FDA-approved for the treatment of melanoma in December 2014, three months after publication of the Phase I data [3]. Approval was based on the results from a dose-comparing, activity-estimating cohort within a multicenter open-label randomized phase $1 \mathrm{~b}$ clinical trial. The major efficacy endpoint was confirmed overall response rate. As a condition of this accelerated approval, Merck was required to conduct a multicenter randomized trial establishing the superiority of pembrolizumab over standard therapy to verify and describe its clinical benefit. These accelerated approvals have led to a substantial increase in the number of clinical trials testing immunotherapies. The efficacy exhibited by checkpoint inhibitors in melanoma patients [4-6] triggered further testing in other 
cancer subtypes such as non-small cell lung cancer [7, 8], renal cell cancer $[9,10]$, bladder cancer [11], and Hodgkin Lymphoma [12]. Other immune-oncology treatment strategies include monoclonal antibodies, cancer vaccines, and adoptive T-cell therapies such as chimeric antigen receptor (CAR)-modified T-cells.

The changing landscape of oncology drug development has resulted in a significant departure from the historical paradigm of a clinical trial design, especially in early development. Phase I trials have served as initial safety studies, with the main objective of identifying the maximum tolerated dose (MTD). The MTD is the highest dose among a range of predefined dose levels that satisfies some safety requirement. The underlying assumption driving the design of a Phase I trial is that both the risk of toxicity and the probability of clinical benefit increase with dose level; thus, the MTD represents the most promising dose for efficacy. The primary toxicity endpoint of interest is most often a binary one, defined in terms of the proportion of patients who experience a dose-limiting toxicity (DLT; yes/no), based on protocol-specific adverse event definitions. The definition of DLT will be defined at the design stage and will differ from trial to trial, depending on disease specification and agent being tested. It typically is characterized by a grade 3 or higher adverse event according to the National Cancer Institute (NCI) Common Terminology Criteria (CTCAE) in the first cycle of treatment. The primary objective is to locate the MTD, defined as highest dose that can be administered to and tolerated by an acceptable proportion of patients. The MTD is often the recommended Phase II doses (RP2D) under the assumption that higher doses are likely to be more effective.

While the traditional approach to Phase I clinical trial designs is adequate for testing cytotoxic agents (e.g., chemotherapy), immune-oncology agents have different assumptions and challenges, some of which may also be applicable to targeted therapies. Thus, trial designs and the corresponding endpoints need to be adapted to the specific agents being investigated [13, 14]. There is a growing need for the implementation of new study designs that address the clinical realities and statistical considerations arising from these new treatment paradigms. In this article, we discuss some of the statistical challenges that immune-oncology agents pose to widelyaccepted methods used in early-phase clinical trials, and make recommendations for implementing innovative trial designs in future studies facing these challenges (Table 1).

\section{Design challenges and recommendations Late-onset toxicities}

Dosing decisions in Phase I trials have traditionally been guided by DLTs that occur in cycle 1 of treatment, which is generally 28 days long. In these trials, MTDs are defined as the highest tolerated dose from cycle 1 , even though patients are administered therapy over the course of several cycles. This approach was appropriate for cytotoxic chemotherapy agents, which generally cause DLTs to be observed early in the treatment course. However, acute toxicity does not provide a complete representation of tolerability for immune-oncology agents. These new agents are administered over extended periods of time, which can result in immune-related adverse events (irAEs) occurring outside of a short-term evaluation window. For example, in a pooled analysis of 576 patients with advanced melanoma who received nivolumab, the median onset time of various treatment-related adverse events of any grade ranged from 5.0 weeks for skin toxicities to 15.1 weeks for renal toxicities [15]. For patients treated with pembrolizumab, the median onset time of irAEs has varied from 1.3 months for hepatitis to 3.5 months for diarrhea [16]. Therefore, the assessment of appropriate doses based solely on DLT definitions from cycle 1 toxicity outcomes is insufficient for immune-oncology therapies. One possible solution to this limitation, which has been utilized in several recently published studies of immune-oncology agents [17-20], is to extend the DLT evaluation window. Additionally, to identify more appropriate doses for further research, there is a growing need to incorporate richer toxicity information beyond DLTs observed in cycle 1 . The most well-known dose-finding method that allows for the incorporation of late toxicities into the design is the time-to-event continual reassessment method (TITECRM) [21]. This method utilizes information from partially observed subjects throughout the trial, without staggering enrollment. In the absence of a DLT, it weights each entered patient by the proportion of the full observation period that he/she has been observed. In the case of no DLTs outside the observation window, the method reduces to the original continual reassessment method (CRM) [22]. Given the availability of $R$ packages $(d f c r m)$ and SAS tools (https://sph.umich.edu/ccb/tite-resources.html) for simulating and conducting the TITE-CRM, the method can be easily tested and implemented into studies in which late-onset DLTs are anticipated. Another recently published method adapts the Bayesian Optimal Interval (BOIN) design to handle late-onset DLTs, and it is accompanied by a user-friendly web application at www.trialdesign.org [23].

\section{More may not be better}

The monotonicity assumption in the traditional Phase I setting is driven by the notion that cytotoxic chemotherapy treatments will directly inhibit the growth of malignant cells, and that the MTD will provide the greatest therapeutic benefit. Conversely, immune-oncology agents generally do not directly impact malignant cells. Instead, immune cells, such as T-cells or natural killer cells, indirectly facilitate the cytotoxic efficacy of these agents. The 


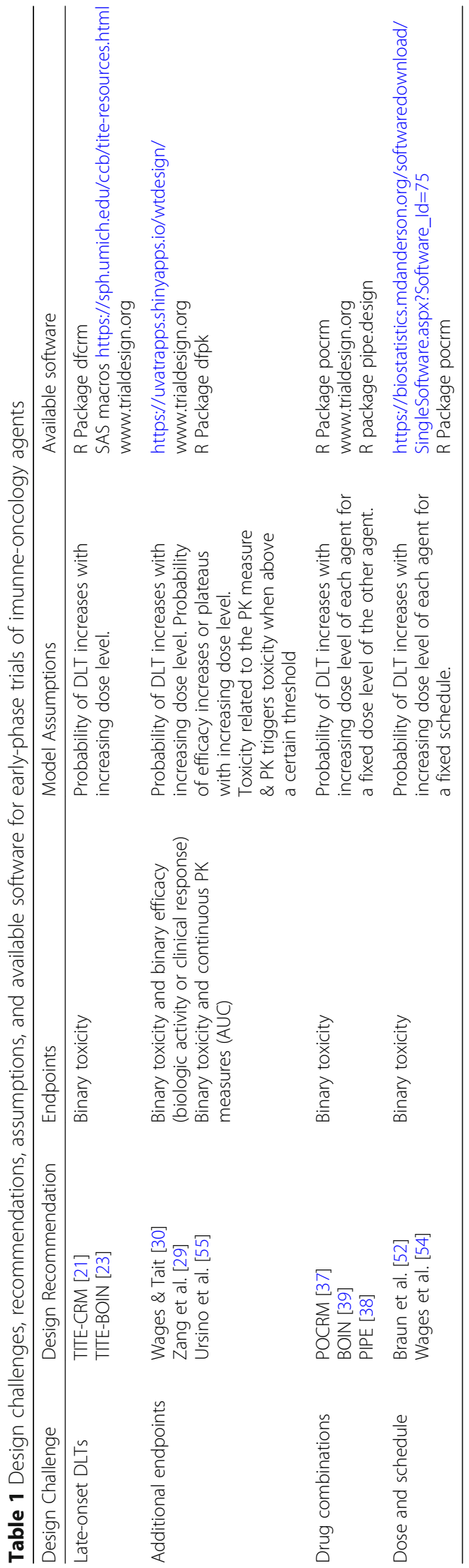

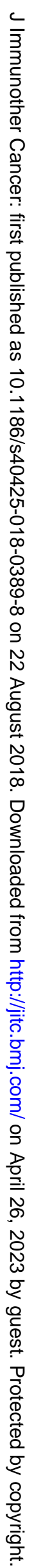


notion that "more is better" for efficacy and "more is worse" for toxicity may not hold true for immune-oncology agents, thus violating the monotonicity assumption that historically underlain Phase I trial designs. Consequently, the early development of immune-oncology agents may need to transition from identifying the MTD to identifying the minimum effective dose. In the case of a dose-efficacy curve that plateaus beyond a certain dose, the optimal dose may fall below the MTD and application of an MTD-based approach could lead to incorrect dosing. For example, in a study of nivolumab, no MTD was reached for doses of 1,3 , and $10 \mathrm{mg} / \mathrm{kg}$ using the original $3+3$ design. However, based on initial signs of activity, additional expansion cohorts were added at doses far below the MTD (0.1 and $0.3 \mathrm{mg} / \mathrm{kg}$ ), indicating a flat dose-efficacy curve among the dose being considered [5]. The dose-toxicity and dose-efficacy relationships for anti-PD-1/PD-L1 therapies remain unclear, with relatively flat toxicity and efficacy rates for doses ranging from 1 to $2 \mathrm{mg} / \mathrm{kg}$ to $20 \mathrm{mg} / \mathrm{kg}$ every 2 or 3 weeks $[17,18,24]$. Conversely, based on results from a study of ipilimumab, patients treated with $10 \mathrm{mg} / \mathrm{kg}$ demonstrated better overall survival than those treated with $3 \mathrm{mg} / \mathrm{kg}$ (15.7 months (95\% confidence interval [CI] 11.6-17.8) versus 11.5 months (95\% CI 9.9-13.3) (hazard ratio [HR] $0.84 ; p=0.04)$ ) [25]. The patients treated with $10 \mathrm{mg} / \mathrm{kg}$ also had a higher rate of irAEs than those treated with $3 \mathrm{mg} / \mathrm{kg}$ [20], indicating dosedependent toxicity and efficacy relationships for ipilimumab. As for toxicity, early-phase data were extensively studied in a review of thirteen Phase I clinical trials of immune checkpoint blocking antibodies [26]. In this review, only one trial identified protocol-defined DLTs [27]. In most of the other trials, the RP2D was based upon the maximum administered dose, which in turn is based on a pre-specified dose range developed from pharmacokinetic data.

Implementation of novel approaches that incorporate multiple endpoints are needed to establish and refine the choice of recommended Phase II doses. In addition to a DLT endpoint, early-phase design strategies should incorporate a tumor activity endpoint, with the goal of identifying an effective dose [7, 28]. Activity markers may include early measures of efficacy (e.g., clinical response), altered pharmacokinetics, altered pharmacodynamic outcomes, and a persistent immune response. The field of immune-oncology often investigates treatments that demonstrate minimal overall toxicity, wherein higher doses may not induce a greater response. The treatment response may increase at low doses and then begin to plateau at higher doses. The goal of the trial then becomes identifying the optimal biologically active dose (OBD), defined as a safe dose that demonstrates the greatest pharmacological activity. In recent years, several new methods have been proposed for identifying the optimal safe and effective dose in Phase
I-II trials [29, 30]. For the problem of locating the optimal biologic dose based on toxicity and activity endpoints in single-agent trials, web applications exist for simulation and implementation of the Wages and Tait [30] method at https://uvatrapps.shinyapps.io/wtdesign/ and for the Zang, Lee, and Yuan [29] method at www.trialdesign.org. One advantage of the Wages and Tait app is the ability of the user to incorporate a stopping rule that terminates the study once a pre-specified maximum number of patients have been accrued to one of the dose levels.

\section{Drug combinations}

It is becoming increasingly popular to treat patients with combination immunotherapy due to the potential for synergistic activity in which the efficacy of both agents together is higher than the efficacy of each agent alone, hopefully without significantly increasing toxicity. The selection of appropriate drug combinations for testing can be very challenging because single agent toxicity data may not be sufficient for characterizing the safety profile of the combination. The most effective and safest doses in drug combinations are seldom the same as those of the individual agents identified in monotherapy trials [31]. Drug combination dose-finding trials also present a greater challenge of finding an MTD combination, or combinations, due to the more complex toxicity and efficacy profiles presented by the potential interaction of the two agents. For example, it is recommended that, combinations of drugs with non-overlapping toxicity profiles be developed whenever possible, since overlapping toxicities can limit escalation of the combination doses to effective levels. In the case of non-overlapping toxicities, the DLT definition of the drug combination is specific to the agents being studied. In practice, Phase I drug combination studies necessitate significant planning at the design stage in order to establish the starting dose of each agent and the total number of combinations to be tested, and these studies can rapidly grow in sample size and cost [32].

In addition to the complexities mentioned above, drug combination studies present additional design challenges to those encountered in single agent studies. Because of the monotonicity assumption, single-agent trials are said to follow a complete order. This is because the ordering of DLT probabilities for any pair of doses is known, and administration of greater doses of the agent can be expected to produce DLT's in increasing proportions of patients. The monotonicity assumption lends itself to escalation along a single line of doses. Given the toxicity response (DLT; yes/no) for a particular patient, either the same dose is recommended for the next patient or the dose is changed to one of two adjacent doses (i.e. either escalate to the next highest dose or de-escalate to the next lowest dose). In studies testing drug combinations, 
the probabilities of DLTs often follow a "partial order" meaning that there are pairs of combinations for which the ordering of the probabilities is unknown. In a multiagent trial, there will most likely be more than one possible treatment with which to treat the next patient cohort in a decision of escalation, and it may not be clear as to which combination to the next cohort should receive.

A traditional approach to this combination dose-finding is to pre-select drug combinations with a known toxicity order and apply a single-agent design by escalating and de-escalating doses along a chosen path [33]. This could be done by, a priori, pre-specifying a subset of combinations for which the toxicity ordering is known. This approach transforms the two-dimensional dose-finding space into a one-dimensional space, and it has been used in much of the early work in dose combinations [34, 35]. The disadvantage of this approach is that it limits the number of dose combinations that can be considered and it can potentially miss promising dose combinations that exist outside of the path. More recent methods have moved away from reducing the two-dimensional dose-finding space to a single dimension, a thorough review of which has been written by Harrington et al. [36]. A number of designs have been proposed for finding the MTD of cytotoxic agents [37-39]. These methods determine combinations to which patients are allocated based solely on toxicity considerations, without accounting for efficacy. As in the single-agent setting, these model-based methods have superior performance to rule-based methods in terms of accuracy of MTD identification, and safety in allocating patients [32]. A web application for the Bayesian Optimal Interval (BOIN) method [39] for combinations is available at www.trialdesign.org, and $R$ packages exist for the partial order continual reassessment method (package pocrm) [37] and the product of independent beta probabilities escalation (PIPE) design (package pipe.design) [38]. The POCRM was successfully implemented in a recently completed, but yet to be published, Phase I trial designed to determine the MTD of a combination of a toll-like receptor (TLR) agonists with or without a form of incomplete Freund's adjuvant (IFA) for the treatment of melanoma (NCT01585350). To our knowledge, the PIPE design has been implemented in two dose-finding studies (NCT02760797, NCT02308072). There are a few existing early-phase designs for drug combination trials that account for both toxicity and efficacy. For example, the method of Wages and Conaway [40] has been adapted and implemented in recently completed and ongoing early-phase studies of combination immune-oncology agents (NCT02126579, NCT02425306) [41, 42] using immunologic response as a binary activity endpoint for driving the design. The $R$ code used to successfully implement these designs are available at http://faculty. virginia.edu/model-based_dose-finding/.
The methods recommended in this section can broadly be implemented in early-phase combination studies that involve immunotherapies in combination with other immunotherapies, or in combination with chemotherapy, radiotherapy, or molecularly targeted agents. Each of these combination types may present their own specific set of trial design challenges, but the methodology described can be generally adapted and applied to meet the research objectives of a broad range of early-phase combination studies. As highlighted at the 2018 ASCO annual meeting, more work is needed in acquiring a better understanding of how to optimally combine therapies [43]. As we learn more, early-phase methodology may need to be adapted to handle unique design challenges associated with novel treatment combinations involving immunotherapies.

\section{Dose and schedule}

The lack of a clear dose-efficacy relationship for both anti-CTLA-4 and anti-PD-1 antibodies has resulted in these agents being assessed at various dose-schedule combinations. For example, ipilimumab was evaluated in four Phase I trials at doses ranging from $3 \mathrm{mg} / \mathrm{kg}$ to $20 \mathrm{mg} / \mathrm{kg}$, without an MTD being identified in any of the trials. A subsequent Phase II trial compared three dose levels of ipilimumab in patients with metastatic melanoma (0.3, 3, and $10 \mathrm{mg} / \mathrm{kg})$; this trial, along with a positive Phase III experience at $3 \mathrm{mg} / \mathrm{kg}$, yielded the registration dose of $3 \mathrm{mg} / \mathrm{kg}$ for 4 cycles [44]. Concurrently, patients with resected melanoma were enrolled in a study using adjuvant ipilimumab at a higher dose $(10 \mathrm{mg} / \mathrm{kg})$ and with an alternative schedule (4 cycles every 3 weeks with maintenance doses every 3 months). This dose and schedule was FDA- approved in the adjuvant setting after it was shown to improve progression free survival [45].

Pembrolizumab has also been studied at different doses $(2 \mathrm{mg} / \mathrm{kg}$ vs $10 \mathrm{mg} / \mathrm{kg})$ and different schedules without a significant difference in efficacy or toxicity by dose or schedule $[3,46]$. More recently, flat dosing of pembrolizumab at $200 \mathrm{mg}$ every 3 weeks has been FDA approved for the treatment of squamous cell carcinoma of the head and neck and PD-L1 positive NSCLC [47, 48]. Further contributing to the uncertainty of dosing design is the use of alternative schedules when immune checkpoint inhibitors are used in combination. For example, the combination of nivolumab $1 \mathrm{mg} / \mathrm{kg}$ and ipilimumab $3 \mathrm{mg} / \mathrm{kg}$ dosed every 3 weeks for 4 doses is FDA-approved for the treatment of metastatic melanoma [49, 50]. Alternative dosing of the combination of nivolumab and ipilimumab was studied in a Phase I trial of patients with metastatic NSCLC in which patients were randomized to receive nivolumab $1 \mathrm{mg} / \mathrm{kg}$ every 2 weeks plus ipilimumab $1 \mathrm{mg} / \mathrm{kg}$ every 6 weeks, nivolumab $3 \mathrm{mg} / \mathrm{kg}$ every 2 weeks plus ipilimumab $1 \mathrm{mg} / \mathrm{kg}$ every 12 weeks, or 
nivolumab $3 \mathrm{mg} / \mathrm{kg}$ every 2 weeks plus ipilimumab $1 \mathrm{mg} / \mathrm{kg}$ every 6 weeks. Response rates and irAEs were similar in the two treatment groups that received nivolumab $3 \mathrm{mg} / \mathrm{kg}$ and both of these arms are considered promising for further study in the randomized Phase 3 trial Checkmate 227 [51].

For these trials, finding an acceptable dose and schedule becomes a two-dimensional dose-finding problem, wherein one dimension is the dose level of the agent and the other dimension is the schedule of therapy. In addressing this type of problem, the approach of Braun et al. [52] based on a time-to-toxicity endpoint, was used to design a dose and schedule finding study (NCT00350818) of de Lima et al. [53]. Available software can be accessed at https://biostatistics.mdanderson.org/softwaredownload/ SingleSoftware.aspx?Software_Id=75. Wages, O'Quigley and Conaway [54] proposed a method for finding a maximum tolerated dose-schedule combination, based on a binary toxicity endpoint, and the $R$ package (pocrm) can be applied to this setting.

\section{Further challenges \\ Novel endpoints in phase I trials}

Under the new assumptions of milder, non-monotone toxicity profiles, determining the OBD is an attractive goal for the early-phase studies. Practically speaking, dose-finding studies incorporating multiple (biological) endpoints have become frequently used approaches for evaluation of targeted, non-cytotoxic drugs. However, there are several barriers that limit their potential to just exploratory endpoints. The inclusion of biological endpoints and determination of an optimal dose based on some biomarker occurrence should rely on pre-specified thresholds such as targeted plasma or blood drug concentration, or other immunologic parameter. Incorporating $\mathrm{PK}$ information in the dose finding process can provide a better estimation of the dose-toxicity curve while maintaining the performance in terms of MTD selection. However, in most phase I trials, the dose-finding and pharmacokinetics (PK) analyses are considered separately, which for small populations might impact the estimation of both the toxicity and PK parameters. Ursino et al. [55] developed and extended methods that take into account PK measurements in sequential Bayesian adaptive early-phase designs. Several models including PK measures either as a covariate or as a dependent variable are examined via simulations in terms of MTD percent correct selection (PCS) and the ability to estimate the dose-response curve. Operating characteristics are presented for a fixed sample size of 30 subjects, six pre-defined dose levels, and seven toxicity scenarios. The main conclusion is that good prior knowledge about PK can help reduce the percentage of overdosing without altering the MTD selection. Still, some of the methods presented (e.g., PKCRM) rely heavily on the choice of PK constraints which in some cases fail to achieve the true MTD. These adaptive pharmacokinetics-based dosefinding designs can be implemented using the $R$ package (dfpk) [56]. Assessing pharmacodynamic (PD) markers as primary endpoints can also be challenging, as they not only require a strong scientific rationale, but also a non-invasive reproducible assay that can track PD markers with minimal harm to the patient [57]. Integration of clinical PK and pre-clinical PD has provided an additional modality of augmenting early clinical data with animal data, but nothing is relevant in the absence of definitive correlations between the target inhibition in PK or PD biomarkers and clinical efficacy (e.g., tumor response).

Adoptive $\mathrm{T}$ cell therapy is a rapidly emerging immunotherapeutic approach that consists of an infusion of genetically engineered $\mathrm{T}$ cells that express a specific antigen on their cell membrane. In 2017, based on a pivotal Phase II trial, the FDA approved the first chimeric antigen receptor (CAR-T) cell therapy (tisagenlecleucel) for children and young adults with B-cell ALL in a population with limited treatment and poor outcomes [58]. With $83 \%$ remission rate, this therapy has demonstrated early and durable response, but much remains to be learned regarding cell proliferation, persistence and mechanisms of relapse. An important predictor of the efficacy of CAR-T cells is their ability to expand in vivo in response to recognition of $\mathrm{CD} 19^{+}$target cells, and therefore, patients that failed to respond in prior studies typically had poor accumulation of CAR-T cells. Interestingly, a recent study investigating CD19 CAR-T cells demonstrated a correlation between cell dose levels (magnitudes of $10^{5}$ cells $/ \mathrm{kg}$ ), earlier/higher peak expansion and clinical response [59]. This finding was also seen in other studies that showed direct correlations between the number of transduced $\mathrm{T}$ cells and antitumor response [60], or correlation between clinical response and persistence of administered cells at one month [61]. Although CD19 CAR-T cells showed a therapeutic effect in patients with relapsed and refractory B-cell ALL, significant toxicities have occurred, especially after infusion of higher CAR-T cell doses. Data imply that an optimal dosing strategy to minimize toxicity would be to initially give low CAR-T cell dose to patients with higher tumor burden, whereas those with low tumor burden may require higher or repeated doses. Thus, under this paradigm shift, dose-finding trials driven solely by toxicity is no longer realistic. Early phase trials should start incorporating more immunological information, while still maintaining acceptable toxicity levels.

The majority of current trials include extended correlative studies, in order to identify promising biomarkers from investigation of immunologic factors of the tumor or tumor microenvironment. Immunologic characteristics 
within the peripheral blood may similarly help predict outcomes following immunotherapy and allow for immunologic monitoring ( $\mathrm{T}$-cell response or percent persistence of transduced T-cells) while on treatment. New dose-finding designs have proposed independent or joint modelling of toxicity and immunological outcomes, both in binary and continuous forms [62]. Also, multi-stage adaptive designs have become more frequent in the early stages of development, with patients being randomized towards doses with higher predicted efficacy. In the context of personalized medicine, immunotherapy is becoming more and more relevant, especially for establishing the patient's immune system profile and developing a tailored treatment/scheduling regimen.

\section{Expansion cohorts}

Phase I trials often include a dose expansion phase with one or more dose expansion cohorts (DEC) after completion of dose escalation with an overall goal to further characterize toxicity, gain preliminary evidence of efficacy, and/or determine the RP2D. It has become common practice that Phase I studies of immuno-oncology agents include multiple DECs based on specific molecular characteristics, biomarker and/or disease type.

The goals of the DEC will drive the sample size of the cohort(s). If the objective is to gain a more precise estimate of the probability of toxicity as has been the conventional purpose of DEC, then expansion cohorts are based on a pre-specified number of patients (e.g., 5, 10, $15)$ treated at MTD. When less than 15 patients are targeted for each DEC, formal sample size justification may not be feasible. The resulting RP2D may differ from the MTD as additional toxicity data are gathered through the expansion phase. In one systematic review, among expansion cohorts with safety objectives, new toxicities were reported in $54 \%$ of trials and the R2PD was modified in 13\% [63]. Re-evaluation of toxicity data after DECs is increasingly important for the safety assessment of checkpoint inhibitors given that adverse events can occur weeks to months after treatment. Approaches for incorporating the additional information include: combining the toxicity data from the initial dose escalation and DEC after all patients have been treated; re-evaluation of the MTD as data from the expansion cohort are obtained with safety stopping rules built-in; evaluation of the MTD incorporating both safety and efficacy; and evaluation of MTD for different sub-populations [64]. Simulation studies have demonstrated that failure to include toxicity outcomes from DECs can result in less accurate estimate of the MTD [63].

Given the advances in technology and in our understanding of tumor biology which have allowed for significantly more drug and drug combinations tested simultaneously, the need for DECs to assess antitumor activity earlier in the drug development has resulted in projected accruals of the DEC to mimic traditional Phase II sample sizes, per cohort $[65,66]$. In this setting, when assessment of efficacy is the primary objective for the DEC, a formal sample size justification with power calculation is recommended along with pre-defined stopping rules for futility to avoid exposing a high number of patients to the risk of ineffective or potentially dangerous treatment.

However, justification of sample sizes can be difficult and has led to very large studies in immune oncology and not always a clear rationale upfront. The anti-PD-L1 compound avelumab is being studied in a Phase I trial with 16 expansion cohorts with a total projected enrollment of 1706 people. In 2011, Merck initiated a first-inhuman trial to determine the safety and recommended dose of pembrolizumab in patients with advanced solid tumors (NCT01295827, NCT01772004) [3]. This Phase I trial ultimately enrolled more than 1200 patients. Impressive response rates and duration of response were observed in patients with metastatic melanoma and nonsmall cell lung cancer, resulting in the addition of DECs to assess efficacy in these two patient populations, evaluate alternative dosing regimens and candidate predictive biomarkers [67]. Although the 1000+ patient Phase I trial is not typical, inclusion of many DECs have become standard and in some ways replacing the traditional clinical trial sequence.

\section{Discussion}

Clinical practice has rapidly incorporated immuneoncologic agents into the standard treatment and management of many cancers. In general, immunotherapy is a treatment modality that activates the immune system to eliminate cancer rather than attacking cancer cells directly. The clinical success of immunotherapy has challenged the existing paradigm for clinical research. With standard chemotherapy or molecularly targeted agents, clinical benefit usually occurs during active treatment and does not persist after treatment discontinuation unlike immunotherapy. Decades of rigorous evaluation through early and late phase clinical trials has informed the understanding and management of the short and long-term effects of toxicities from chemotherapy. However, less is known regarding the toxicities associated with immunotherapy.

Due to the activation of the immune system, immuneoncologic agents can inadvertently activate the immune system against self, resulting in significant immune-related adverse events [68]. Since serious immune-related adverse events represent immune activation, they may actually reflect therapeutic benefit. Furthermore, while low grade immune-related adverse events are managed with dose reductions, management of more severe adverse events 
includes administration of anti-inflammatory therapies such as corticosteroids, infliximab or mycophenolate to dampen immune activation $[68,69]$, potentially weakening therapeutic benefit.

Understanding the mechanisms of response and adverse events in the context of these agents is critical for the selection of appropriate clinical trial designs. According to the Institute for Clinical Immuno-Oncology between 2006 and 2014, the number of clinical trials registered on ClinicalTrials.gov increased from 9321 to 18,400 [70]. According to the "Medicines in Development for Immuno-Oncology 2017 Report," from PhRMA in partnership with the American Cancer Society Action Network (ASCAN), more than 248 new immuno-oncologic agents are currently in clinical trials or awaiting U.S. FDA review [71]. In addition, from 2015 to 2017, the number of combination studies listed on ClinicalTrials.gov combining PD-1 or PD-L1 inhibitors with other therapies has more than tripled from 215 to 765 (combination trials with pembrolizumab [ $n=268]$; nivolumab $[n=242]$; durvalumab [ $n=123]$; atezolizumab [ $n=83]$; avelumab [ $n=18]$; and others $[n=49]$ ] [72]. Implementation of innovative design strategies in the early development of combination immunotherapies is critical to delivering more effective therapies with improved outcomes.

In this paper, we reviewed design considerations for early phase clinical trials of immuno-oncology agents and when available provided reference to software for applicability of these designs. There is increasing demand for study designs that are best suited and optimal in this setting. A deeper understanding of clinically meaningful endpoints, characterization of toxicity, identification of immune parameters and mutational burden to help guide patient selection will allow for the further development of novel designs for early phase trials of immuno-oncologic agents.

\begin{abstract}
Abbreviations
BOIN: Bayesian optimal interval design; CAR: Chimeric antigen receptor; CRM: Continual reassessment method; CTCAE: Common Terminology Criteria for Adverse Events; CTLA4: Cytotoxic T-lymphocyte antigen-4; DEC: Dose expansion cohort; DLT: Dose limiting toxicity; FDA: Food and Drug Administration; FDASIA: Food and Drug Administration Safety and Innovation Act (FDASIA); IFA: Incomplete Freund's adjuvant; irAEs: Immune-related adverse events; MTD: Maximum tolerated dose; NCl: National Cancer Institute; NSCLC: Non-small cell lung cancer; OBD: Optimal biologically active dose; PD: Pharmacodynamics; PD-1: Programmed cell death protein-1; PD-L1: Programmed death ligand-1; PIPE: Product of independent probabilities escalation; PK: Pharmacokinetic: POCRM: Partial order continual reassessment method; RP2D: recommended phase II dose; TITE-BOIN: Time-to-event Bayesian optimal interval design; TITE-CRM: Time to event continual reassessment method; TLR: Toll-like receptor
\end{abstract}

\section{Acknowledgements}

The authors would like to thank two reviewers for helpful comments that helped us improve the original submission.

\section{Funding}

Dr. Wages is supported by National Cancer Institute award number K25CA181638. Dr. Chiuzan is supported by National Center for Advancing Translational Sciences grant number UL1TR001873. Dr. Panageas is supported by Memorial Sloan
Kettering Cancer Center Core Grant (P30CA008748). These funding bodies did not have any role in the design of this study, collection, analysis, and interpretation of data, nor in the writing of the manuscript.

\section{Availability of data and materials}

Not applicable.

\section{Authors' contributions}

All authors contributed to drafting and revising the manuscript. All authors gave final approval of the manuscript submitted for publication.

\section{Ethics approval and consent to participate}

Not applicable. The research contained in this manuscript does not involve human subjects, human material, or human data.

\section{Consent for publication}

Not applicable.

\section{Competing interests}

The authors declare that they have no competing interests.

\section{Publisher's Note}

Springer Nature remains neutral with regard to jurisdictional claims in published maps and institutional affiliations.

\section{Author details}

'Division of Translational Research \& Applied Statistics, Department of Public Health Sciences, University of Virginia, P.O. Box 800717, Charlottesville, VA, USA. ${ }^{2}$ Department of Biostatistics, Mailman School of Public Health, Columbia University, New York, NY, USA. ${ }^{3}$ Department of Epidemiology \& Biostatistics, Memorial Sloan Kettering Cancer Center, New York, NY, USA.

Received: 6 April 2018 Accepted: 12 July 2018

Published online: 22 August 2018

\section{References}

1. Food and Drug Administration Safety and Innovation Act, S 3187, $112^{\text {th }}$ Cong, 2012.

2. Food and Drug Administration. Guidance for Industry: Expedited Programs for Serious Conditions - Drugs and Biologics. Washington, DC: Food and Drug Administration, US Dept of Health and Human Services; 2014.

3. Robert C, Ribas A, Wolchok JD, Hodi FS, Hamid O, Kefford R, Weber JS, Joshua AM, Hwu WJ, Gangadhar TC, Patnaik A, Dronca R, Zarour H, Joseph RW, Boasberg P, Chmielowski B, Mateus C, Postow MA, Gergich K, ElassaissSchaap J, Li XN, lannone R, Ebbinghaus SW, Kang SP, Daud A. Antiprogrammed-death-receptor-1 treatment with pembrolizumab in ipilimumab-refractory advanced melanoma: a randomised dose-comparison cohort of a phase 1 trial. Lancet. 2014;384:1109-17.

4. Hodi FS, O'Day SJ, McDermott DF, et al. Improved survival with ipilimumab in patients with metastatic melanoma. N Engl J Med. 2010;363:711-23.

5. Robert $C$, Thomas $L$, Bondarenko I, et al. Ipilimumab plus dacarbazine for previously untreated metastatic melanoma. N Engl J Med 2011;364:2517-26.

6. Hamid O, Robert C, Daud A et al. Safety and tumor responses with lambolizumab (anti-PD-1) in melanoma. N Engl J Med. 2013; 369:134-44.

7. Gettinger SN, Horn L, Gandhi L, et al. Overall survival and long-term safety of Nivolumab (anti-programmed death 1 antibody, BMS-936558, ONO-4538) in patients with previously treated advanced non-small-cell lung Cancer. J Clin Oncol. 2015:33:2004-12.

8. Garon EB, Rizvi NA, Hui R, et al. Pembrolizumab for the treatment of nonsmall-cell lung Cancer. N Engl J Med. 2015;372:2018-28.

9. Motzer RJ, Rini BI, McDermott DF, et al. Nivolumab for metastatic renal cell carcinoma: results of a randomized phase II trial. J Clin Oncol. 2015;33:1430-7.

10. Motzer RJ, Escudier B, McDermott DF, et al. Nivolumab versus Everolimus in advanced renal-cell carcinoma. N Engl J Med. 2015;373:1803-13.

11. Rosenberg JE, Hoffman-Censits J, Powles T, et al. Atezolizumab in patients with locally advanced and metastatic urothelial carcinoma who have progressed following treatment with platinum-based chemotherapy: a single-arm, multicentre, phase 2 trial. Lancet. 2016;387:1909-20.

12. Ansell SM, Lesokhin AM, Borrello l, et al. PD-1 blockade with nivolumab in relapsed or refractory Hodgkin's lymphoma. N Engl J Med. 2015;372:311-9. 
13. Chiuzan C, Garrett-Mayer E, Yeats S. A likelihood-based approach for computing the operating characteristics of the $3+3$ phase I clinical trial design with extensions to other a+B designs. Clin Trials. 2015;12:24-33.

14. Chiuzan C, Shtaynberger J, Manji GA, Duong JK, Schwartz GK, Ivanova A, Lee SM. Dose-finding designs for trials of molecularly targeted agents and immunotherapies. J Biopharm Stat. 2017;27:477-94.

15. Weber JS, Hodi S, Wolchok JD, et al. Safety profile of nivolumab monotherapy: a pooled analysis of patients with advanced melanoma. J Clin Oncol. 2017;35:785-92

16. Opdivo (R) [package insert]. New York, NY: Bristol-Myers Squibb; 2014.

17. Topalian SL, Hodi FS, Brahmer JR, et al. Safety, activity, and immune correlates of anti-PD-1 antibody in cancer. N Engl J Med. 2012;366:2443-54.

18. Brahmer JR, Tykodi SS, Chow LQM, et al. Safety and activity of anti-PD-L1 antibody in patients with advanced cancer. N Engl J Med. 2012;366:2455-65.

19. Lutzky J, Antonia S, Blake-Haskins A, et al. A phase 1 study of MEDI4736, an anti-PD-L1 antibody, in patients with advanced solid tumors. J Clin Oncol. 2014:32:5s. abstr 3001

20. Wolchok JD, Kluger H, Callahan MK, et al. Nivolumab plus ipilimumab in advanced melanoma. N Engl J Med. 2013;369:122-33.

21. Cheung YK, Chappell R. Sequential designs for phase I clinical trials with late-onset toxicities. Biometrics. 2000;56:1177-82.

22. O'Quigley J, Pepe M, Fisher L. Continual reassessment method: a practical design for phase I clinical trials in cancer. Biometrics. 1990:46:33-48.

23. Yuan Y, Lin R, Li D, Nie L, Warren K. Time-to-event Bayesian optimal interval design to accelerate phase I trials. Clin Cancer Res 2018; [epub ahead of print] doi: https://doi.org/10.1158/1078-0432.CCR-18-0246.

24. Patnaik A, Kang SP, Rasco D, et al. Phase I study of pembrolizumab (MK3475; anti-PD-1 monoclonal antibody) in patients with advanced solid tumors. Clin Cancer Res. 2015;21:4286-93.

25. Ascierto PA, Del Vecchio M, Robert C, et al. Overall survival (OS) and safety results from a phase 3 trial of ipilimumab (IPI) at $3 \mathrm{mg} / \mathrm{kg}$ vs $10 \mathrm{mg} / \mathrm{kg}$ in patients with metastatic melanoma (MEL). Ann Oncol 2016; 27(suppl 6): 379-400.

26. Postel-Vinay S, Aspeslagh S, Lanoy E, et al. Challenges of phase 1 clinical trials evaluating immune checkpoint-targeted antibodies. Ann Oncol. 2015;27:214-24

27. Ribas A, Camacho LH, Lopez-Berestein $\mathrm{G}$, et al. Antitumor activity in melanoma and anti-self responses in a phase I trial with the anti-cytotoxic $T$ lymphocyte-associated antigen 4 monoclonal antibody CP-675,206. J Clin Oncol. 2015;23:8968-77.

28. Sachs JR, Mayawala K, Gadamsetty S, Kang SP, de Alwis DP. Optimal dosing for targeted therapies in oncology: drug development cases leading by example. Clin Cancer Res. 2016;22:1318-24.

29. Zang Y, Lee JJ, Yuan Y. Adaptive designs for identifying optimal biological dose for molecularly targeted agents. Clin Trials. 2014;11:320-7.

30. Wages NA, Tait C. Seamless phase I/II adaptive design for oncology trials of molecularly targeted agents. J Biopharm Stat. 2015;25:903-20.

31. Wong KM, Capasso A, Eckhardt G. The changing landscape of phase I trials in oncology. Nat Rev Clin Oncol. 2016;13:106-17.

32. Riviere MK, Le Tourneau C, Paoletti $X$, et al. Designs of drug-combination phase I trials in oncology: a systematic review of the literature. Ann Oncol. 2015;26:669-74.

33. Mandrekar SJ. Dose-finding trial designs for combination therapies in oncology. J Clin Oncol. 2014;32:65-7.

34. Korn EL, Simon R. Using the tolerable-dose diagram in the design of phase I combination chemotherapy trials. J Clin Oncol. 1993:11:794-801.

35. Kramar A, Lebecq A, Candalh E. Continual reassessment methods in phase I trials of the combination of two agents in oncology. Stat Med. 1999;18:1849-64.

36. Harrington JA, Wheeler GM, Sweeting MJ, Mander AP, Jodrell DI. Adaptive designs for dual-agent phase I dose-escalation studies. Nat Rev Clin Oncol. 2013;10:277-88.

37. Wages NA, Conaway MR, O'Quigley J. Dose-finding design for multi-drug combinations. Clin Trials. 2011:8:380-9.

38. Mander AP, Sweeting MJ. A product of independent beta probabilities dose escalation design for dual-agent phase I trials. Stat Med. 2015;34:1261-76.

39. Lin R, Yin G. Bayesian optimal interval design for drug combination trials. Stat Methods Med Res. 2015:26:2155-67.

40. Wages NA, Conaway MR. Phase I/II adaptive design for drug combination oncology trials. Stat Med. 2014;33:1990-2003.

41. Wages NA, Slingluff CL, Jr, Petroni GR. A phase I/II adaptive design to determine the optimal treatment arm from a set of combination immunotherapies in high-risk melanoma. Contemporary Clinical Trials 2015; 41: 172-179.
42. Wages NA, Slingluff CL, Petroni GR. Statistical controversies in clinical research: early-phase adaptive design for combination immunotherapies. Ann Oncol. 2017;28:697-701.

43. Much Work Remains to Determine Optimal Immunotherapy Combinations. https:/am.asco.org/much-work-remains-determine-optimal-immunotherapycombinations? $=40342873 \&$ sfmc sub $=1758226242 \&$ l $=18416124$ HTML \&u=709159 599\&mid=10831468\&jb=153\&et_cid=40342873\&et_rid=1758226242\&linkid=Much +Work+Remains+to+Determine+Optimal+Immunotherapy+Combinations. Accessed 8 June 2018

44. Wolchok JD, Neyns B, Linette $G$, et al. Ipilimumab monotherapy in patients with pretreated advanced melanoma: a randomised, double-blind, multicentre, phase 2, dose-ranging study. Lancet Oncol. 2010;11:155-64.

45. Eggermont AMM, Chiarion-Sileni V, Grob J-J, et al. Adjuvant ipilimumab versus placebo after complete resection of high-risk stage III melanoma (EORTC 18071): a randomised, double-blind, phase 3 trial. Lancet Oncol. 2015;16:522-30.

46. Robert C, Schachter J, Long GV, et al. Pembrolizumab versus ipilimumab in advanced melanoma. N Engl J Med. 2015;372:2521-32.

47. Reck M, Rodríguez-Abreu D, Robinson AG, et al. Pembrolizumab versus chemotherapy for PD-L1-positive non-small-cell lung Cancer. N Engl J Med. 2016;375:1823-33.

48. Seiwert TY, Burtness B, Mehra R, et al. Safety and clinical activity of pembrolizumab for treatment of recurrent or metastatic squamous cell carcinoma of the head and neck (KEYNOTE-012): an open-label, multicentre, phase 1b trial. Lancet Oncol. 2016;17:956-65.

49. Larkin J, Chiarion-Sileni V, Gonzalez R, et al. Combined nivolumab and ipilimumab or monotherapy in untreated melanoma. N Engl J Med. 2015;373:23-34

50. Postow MA, Chesney J, Pavlick AC, et al. Nivolumab and ipilimumab versus ipilimumab in untreated melanoma. N Engl J Med. 2015;372:2006-17.

51. Hellmann MD, Rizvi NA, Goldman JW, et al. Nivolumab plus ipilimumab as first-line treatment for advanced non-small-cell lung cancer (CheckMate 012): results of an open-label, phase 1. multicohort study Lancet Oncol. 2017; 18:31-41.

52. Braun $T M$, Thall $P F$, Nguyen $H Q$, de Lima $M$. Simultaneously optimizing dose and schedule of a new cytotoxic agent. Clin Trials. 2007;4:113-24.

53. de Lima M, Giralt S, Thall PF, et al. Maintenance therapy with low dose azacitidine after allogenic hematopoietic stem cell transplantation for recurrent acute myelogenous leukemia or myelodysplastic syndrome: a dose and schedule finding study. Cancer. 2010;116:5420-31.

54. Wages NA, O'Quigley J, Conaway MR. Phase I design for completely or partially ordered treatment schedules. Stat Med. 2013;33:569-79.

55. Ursino M, Zohar S, Lentz, et al. dose-finding methods for phase I clinical trials using pharmacokinetics in small populations. Biom J. 2017;59:804-25.

56. Toumazi A, Comets E, Alberti C, et al. dfpk: an R-package for Bayesian dosefinding designs using pharmacokinetics (PK) for phase I clinical trials. Computer Methods Programs Biomed. 2018;157:163-77.

57. Cook N, Hansen AR, Siu LL, Abdul Razak AR. Early phase clinical trials to identify optimal dosing and safety. Mendelsohn J, Ringborg U, Schilsky R, eds. Mol Oncol 2015: 9: 997-1007.

58. Maude SL, et al. Tisagenlecleucel in children and young adults with B-cell lymphoblastic leukemia. N Engl J Med. 2018:378:439-48.

59. Turtle CJ, Hanafi L-A, Berger C, et al. CD19 CAR-T cells of defined CD4 ${ }^{+}$: CD8 ${ }^{+}$composition in adult B cell ALL patients. J Clin Invest. 2016:126:2123-38.

60. Gattinoni L, Klebanoff CA, Palmer DC, et al. Acquisition of full effector function in vitro paradoxically impairs the in vivo antitumor efficacy of adoptively transferred CD8+ T cells. J Clin Invest. 2005;115:1616-26.

61. Johnson LA, Morgan RA, Dudley ME, et al. Gene therapy with human and mouse T-cell receptors mediates cancer regression and targets normal tissues expressing cognate antigen. Blood. 2009;114:535-46.

62. Chiuzan C, Garrett-Mayer E, Nishimura M. An adaptive dose-finding design based on both safety and immunologic responses in cancer clinical trials. Stat in Biopharm Res. 2018; in press

63. Manji A, Brana I, Amir E, et al. Evolution of clinical trial Design in Early Drug Development: systematic review of expansion cohort use in single-agent phase I Cancer trials. J Clin Oncol. 2013;31:4260-7.

64. lasonos A, O'Quigley J. Design considerations for dose-expansion cohorts in phase I trials. J Clin Oncol. 2013;31:4014-21.

65. Iasonos A, O'Quigley J. Clinical trials: Early phase clinical trials-are dose expansion cohorts needed? Nat Rev Clin Oncol. 2015;12:626-8.

66. lasonos A, O'Quigley J. Dose expansion cohorts in phase I trials. Stat Biopharm Res. 2016;8:161-70. 
67. Prowell TM, Theoret MR, Pazdur R. Seamless oncology-drug development. N Engl J Med. 2016;374:2001-3.

68. Weber JS, Yang JC, Atkins MB, Disis ML. Toxicities of immunotherapy for the practitioner. J Clin Oncol. 2015;33:2092-9.

69. Friedman CF, Proverbs-Singh TA, Postow MA. Treatment of the immunerelated adverse events effects of immune checkpoint inhibitors: a review. JAMA Oncol. 2016;2:1346-53.

70. Ehrhardt S, Appel LJ, Meinert CL. Trends in National Institutes of Health funding for clinical trials registered in ClinicalTrials.gov. JAMA. 2015;314:2566-7.

71. Pharmaceutical Research and Manufacturers of America, The American Cancer Society Cancer Action Network. Medicines in Development 2017 Report: Immuno-oncology. 2017; http://www.phrma.org/medicines-indevelopment-immuno-oncology.

72. Plieth J, Elmhirst E. PD-1/PD-L1 combination therapies. 2017; www. evaluategroup.com/PD1-2017.

Ready to submit your research? Choose BMC and benefit from:

- fast, convenient online submission

- thorough peer review by experienced researchers in your field

- rapid publication on acceptance

- support for research data, including large and complex data types

- gold Open Access which fosters wider collaboration and increased citations

- maximum visibility for your research: over $100 \mathrm{M}$ website views per year

At $\mathrm{BMC}$, research is always in progress.

Learn more biomedcentral.com/submissions 